\title{
Kein Tarifschutz für freie Arztwahl am Spital, Zusatzrechnung zulässig
}

\author{
Auszug aus dem Bundesgerichtsentscheid 5P.74/2004 vom 31. August 2004 in Sachen Helsana AG gegen Sanitäts- \\ departement Basel-Stadt
}

$[\ldots]$

2.2 Hinsichtlich des Zuschlags von 120 bis $170 \%$ für die Arzthonorare bei stationären Privatpatienten macht die Beschwerdeführerin geltend, ärztliche Leistungen laut Spitalleistungskatalog (Pos. 1000-3999 SLK bzw. bei fehlenden SLKPositionen nach entsprechenden TARMED-Positionen) stellten Pflichtleistungen nach dem KVG dar, die vollumfänglich vom Tarifschutz erfasst würden. Indes nennt die Beschwerdeführerin, indem sie die freie Arztwahl erwähnt, auch hier die echte Mehrleistung gleich selbst:

Das KVG garantiert innerhalb örtlicher Grenzen jedem Versicherten die freie Wahl des Leistungserbringers (vgl. Art. 41 KVG; BBl 1992 I 168f.). Hingegen statuiert es, abgesehen von Notfällen, keine Behandlungspflicht für Ärzte, weshalb im stationären Bereich faktisch nur Patienten mit einer Zusatzversicherung die freie Arztwahl haben (Conti, Zusatzhonorar des Arztes und KVG, in: AJP 2001, S. 1148ff., insb. S. 1152). Privatpatienten wünschen sich regelmässig die Behandlung durch einen Chef- oder Belegarzt. Das Sanitätsdepartement weist in seiner Vernehmlassung denn auch zutreffend darauf hin, dass die Beschwerdeführerin mit ebendiesem Argument für den Abschluss ihrer Zusatzversicherung (privat) wirbt.

Die freie Arztwahl für Zusatzversicherte im stationären Bereich führt dazu, dass Chefärzte für die Spitalträger mit entsprechenden Kostenfolgen - insbesondere bei den Privatpatienten zahlreiche Leistungen erbringen, für die sie eigentlich überqualifiziert sind, sei es, weil sie bei allgemeinversicherten Patienten einfachere Behandlungen von vornherein nicht selbst durchführen würden, sei es, dass sie im Vorfeld und im Nachgang zu einer komplizierten Behandlung, die als solche einen Spezialisten erfordert, zahlreiche Handlungen persönlich vornehmen, die im Normalfall an Assistenzärzte oder das Pflegepersonal delegiert würden. Es darf zudem als notorisch bezeichnet werden, dass Privatpatienten andere Erwartungen an den behandelnden Arzt stellen und dieser beispielsweise häufiger als medizinisch indiziert Sprechstunden abhält oder Visitationen vornimmt (vgl. zum Ganzen: Kie- ser, Die Bedeutung des krankenversicherungsrechtlichen Tarifschutzes im stationären Bereich, in: SZS 2003, S. 419ff., insb. S. 427; Kuhn, $\mathrm{Zu}$ Zusatzrechnungen bei ärztlichen Zusatzleistungen, in: SÄZ 2000, S. 77ff., insb. S. 80).

Die freie Arztwahl im stationären Bereich stellt damit eine ganz erhebliche Mehrleistung dar, die weit über die obligatorische Krankenversicherung hinausgeht. Der Tarifschutz gemäss Art. 44 KVG beschränkt sich hier darauf, dass der Versicherer nach KVG jene Kosten übernehmen muss, welche sich ergeben würden, wenn der Versicherte in der allgemeinen Abteilung behandelt worden wäre (sog. Austauschbefugnis; vgl. Maurer, Das neue Krankenversicherungsrecht, Basel 1996, S. 81 unten; Conti, a.a.O., S. 1152). Ein Honorarzuschlag bei stationären Privatpatienten ist somit gerechtfertigt, zumal Privatpatiententarife im stationären Bereich die Rechte und Behandlungsmöglichkeiten der allgemeinversicherten Personen nicht beeinträchtigen (vgl. Eugster, Krankenversicherung, in: Schweizerisches Bundesverwaltungsrecht, Loseblattsammlung, Basel 1998, N. 325 und Fn. 785). Entgegen der Behauptung der Beschwerdeführerin bedarf die erwähnte Mehrleistung in der Tarifordnung auch keiner näheren Umschreibung, darf doch ihr Inhalt - wie der Anspruch auf ein Einzelzimmer bei den Tagesteilpauschalen - als allgemein bekannt vorausgesetzt werden.

2.3 Auch für den ambulanten Bereich hat das Bundesgericht in BGE 126 III 345 E. 3b S. 350 eine Abrechnung ausserhalb des festgesetzten Tarifs zugelassen, wenn es um die Vergütung echter Mehrleistungen geht, die über den Leistungsumfang der obligatorischen Krankenpflegeversicherung hinausgehen. Es hat jedoch betont, dass es sich um echte Mehrleistungen handeln müsse, die ein «Plus» darstellten (vgl. die dort genannten Beispiele; für weitere Beispiele vgl. namentlich: Wohnlich, Zusatzleistungen im ambulanten Bereich der Krankenversicherung, Diss. Zürich 2002, S. 54ff.).

Wenn sich die Beschwerdeführerin auf diese Rechtsprechung beruft, übersieht sie, dass in der angefochtenen Tarifordnung für den Honorar- 
zuschlag bei ambulanten Behandlungen die Tarifschutzbestimmungen des KVG und UVG vorbehalten sind. Ihre Rüge, die Tarifordnung sei diesbezüglich nicht mit Art. 44 KVG vereinbar, stösst deshalb ins Leere. Das Sanitätsdepartement führt in seiner Vernehmlassung denn auch aus, dass der 50\%ige Honorarzuschlag auf Grund des Vorbehalts selbstverständlich nur für Perso- nen gelten könne, die von ihrem Wohnsitz her bei Behandlungen in der Schweiz nicht dem Geltungsbereich des KVG unterstünden.

\section{$[\ldots]$}

Demnach erkennt das Bundesgericht: Die Beschwerde wird abgewiesen.

TARMED: Anpassung des Taxpunktwertes in den Bereichen Unfallversicherung, Militärversicherung und Invalidenversicherung

Die Assessment-Kommission hat eine Senkung des Taxpunktwertes von Fr. 1.- auf Fr. -.92 beschlossen.

Die Assessment-Kommission ist die von den Vertragsparteien eingesetzte Kommission, die die Einhaltung Fallkostenstabilität im Bereich der freipraktizierenden Ärzteschaft überwacht. Sie beurteilt monatlich die mittleren Fallkosten der Unfallversicherer, der Militärversicherung und der Invalidenversicherung, und zwar global und auch in bezug auf die einzelnen Fachgruppen.

Gestützt auf die ermittelte Kostenentwicklung per Ende der fallkostenstabilen Phase und die getroffenen Vereinbarungen wird nun der Taxpunktwert per 1. November 2004 gesamtschweizerisch auf 92 Rappen korrigiert. Dieser Wert gilt für die freipraktizierende Ärzteschaft im Bereich der eidgenössischen Sozialversicherer (UV/MV/IV).

Die Medizinaltarif-Kommission UVG (für die obligatorische Unfallversicherung), die Militärversicherung, die Invalidenversicherung und auch die Verbindung der Schweizer Ärztinnen und Ärzte FMH stützen den Beschluss der Assessment-Kommission.

Für die Medizinaltarif-Kommission:

Für die FMH:

Beat Huwiler,

Zentralstelle für Medizinaltarife UVG

Dr. med. François Bossard, Präsident Assessment-Kommission

TARMED: adaptation de la valeur du point tarifaire pour l'assurance-accidents, I'assurance militaire et l'assurance-invalidité

La Commission d'évaluation a décidé de baisser la valeur du point tarifaire de Fr. 1.à Fr. -.92 .

La Commission d'évaluation (en allemand «Assessment-Kommission») est l'organe chargé par les parties à la convention de surveiller le respect de la stabilité des coûts chez les médecins en pratique privée. Chaque mois, cette commission examine les coûts par cas moyens supportés par les assureurs-accidents ainsi que par l'assurance militaire et l'assurance-invalidité et ce, tant de manière globale qu'en fonction des différentes disciplines médicales.

Vu l'évolution des coûts constatée à la fin de la période de stabilité des coûts par cas et en vertu des accords conclus, la valeur du point tarifaire sera corrigée et fixée à 92 centimes dès le $1^{\text {er }}$ novembre 2004. Cette valeur du point s'applique aux médecins libres praticiens fournissant des prestations dans le cadre des assurances sociales fédérales (AA, $\mathrm{AI}, \mathrm{AM}$ ).

La Commission des tarifs médicaux LAA (représentant l'assurance-accidents obligatoire), l'assurance militaire et l'assurance-invalidité ainsi que la Fédération des médecins suisses (FMH) ont donné leur aval à la décision prise par la Commission d'évaluation.

Pour la Commission des tarifs médicaux LAA: Pour la FMH:

Beat Huwiler,

Service central des tarifs médicaux LAA

Dr François Bossard, président de la Commission d'évaluation 